\title{
A Study into the Dynamics of Three Dimensional Tape Spring Folds
}

\author{
Scott J.I. Walker* and Guglielmo Aglietti ${ }^{\dagger}$ \\ School of Engineering Sciences, University of Southampton, UK
}

\begin{abstract}
One of the most significant drivers in satellite design is the minimization of mass, in the attempt to reduce the large costs involved in the launch. With technological advances across many fields it is now widely known that very low mass satellites can perform a wide variety of missions. However, the satellite power requirement does not reduce linearly with mass, creating the need for efficient and reliable small satellite deployable structures. One structural solution for this application is tape springs. Tape springs have been previously studied by many countries for space applications focusing on two dimensional systems. This work studies the possible impact of using tape springs folded in three dimensions. By initially analytically determining the static moments created, simple deployment models can be constructed for tape springs in free space. By determining the impact of these moments about an array fold line, a dynamic model of an array can be created which is directly comparable to the two dimensional system. The impact of the three dimensional fold can then be determined.
\end{abstract}

\section{Nomenclature}

$L \quad$ Total tape spring length

E Young's Modulus

$D \quad$ Flexural Rigidity

$\nu \quad$ Poisson's Ratio

$\alpha \quad$ Angle subtended by cross-section of spring

$a \quad$ Width of un-deformed tape spring

$t \quad$ Thickness of the tape spring

$\theta \quad$ Total fold angle

$\gamma \quad$ Total twist angle

$M \quad$ Bending moment applied to the strip (about y axis)

$M_{+}^{\max } \quad$ Peak moment for opposite sense bending

$M_{-}^{\max } \quad$ Peak moment for equal sense bending

$M_{+}^{*}, M_{-}^{*}$ Steady state moment for opposite and equal sense bending respectively

$T \quad$ Torsional moment of tape spring

$R_{x}, R_{y} \quad$ Initial longitudinal and transverse radii of curvature respectively

$\kappa_{x, 0}, \kappa_{y, 0}$ Initial longitudinal and transverse curvatures respectively

$\kappa_{x} \quad$ Change in longitudinal curvature

$\kappa_{x y, 0} \quad$ Initial twisting curvature

$\kappa_{x y} \quad$ Change in twisting curvature

$\bar{M}, \bar{T}, \bar{\kappa} \quad$ Non dimensional, moment, torque and curvature of the tape spring

$\lambda_{1}, \lambda_{2} \quad$ Curvatures along lines of principle curvatures

$\phi \quad$ Relative angle of lines of principle curvature

$m_{A}, m_{B}$ Initial constants relating $M$ and $\theta$

$I_{y} \quad$ Inertia of tape spring around y axis

*Graduate Student, Astronautics Research Group

${ }^{\dagger}$ Lecturer in Aerospace Structural Dynamics, Astronautics Research Group

Copyright (c) 2003 by Scott J.I. Walker. Published by the American Institute of Aeronautics and Astronautics, Inc., with permission.

\author{
$m_{C}, m_{D}$ Initial constants of dynamic equation \\ $A_{m}, B_{m}$ Constants for tape spring dynamic solution \\ $C_{m}, \lambda_{i}$ Constants for tape spring dynamic solution \\ $M_{\text {hinge }}$ Moment about array hinge line \\ $\mu \quad$ Angle of tape spring from the \\ perpendicular of the array fold line \\ $l_{1}, l_{2} \quad$ Array lengths defined from $\mathrm{L}$ and $\mu$ \\ $\Delta \quad$ Angle of deployed array \\ $\Lambda \quad$ Opposite angle of $\Delta(=\pi-\Delta)$ \\ $\varepsilon \quad$ Angle from array vertical $\left(=\Delta-\frac{\pi}{2}\right)$ \\ $\beta \quad$ Angle of plane (which contains the tape \\ $\sigma \quad$ Opposite angle of $\theta(=\pi-\theta)$ \\ $I_{\text {array }} \quad$ Inertia of unfolding array
}

\section{Introduction}

7 HE total satellite mass has always been a key 1 parameter in satellite design and with advances in micro technology ${ }^{1}$ there is an increasing interest in the space industry to develop very low mass satellites for a wide variety of applications. Following research in micro ion propulsion systems for attitude control ${ }^{2}$ and the miniaturization of ground based mobile communications, it is known that the satellite power requirement does not reduce linearly with mass. Many small satellite missions simply use body-mounted photovoltaic cells, severely restricting the total available power (due to the small surface area of the satellite). With further reductions in the mass and hence the surface area of the satellite, the total power achievable from body-mounted cells reduces. This creates the need for small satellite, area deployment devices. Some small satellites have used simple fold out panels to increase the projected area to the sun. Other solutions have been suggested such as the Powersphere concept,${ }^{3}$ developed from inflatable technology, that encases the 
nano satellite projecting a constant area to the sun at all attitudes. With any small satellite deployable system, the key is to create a supporting structure with the required stiffness, of minimum mass and high deployment reliability. Two possible structural solutions that currently exist for these applications are inflatable structures and tape springs. This study focuses on the dynamic deployment of tape springs folded in three dimensions.

\section{Background}

Tape springs are defined as thin metallic strips with a curved cross section, which have the key property that they are continuous (i.e. contains no mechanical hinges or other folding devices) and yet can still be folded elastically. ${ }^{4}$ They do not require any lubrication and automatically lock in the deployed configuration. In recent years there has been committed research into the use of tape springs in deployable structures. Such work has focused on tape springs folded in two dimensions, as shown in figure 1 (folded through $\theta$ degrees). This research has resulted in the development

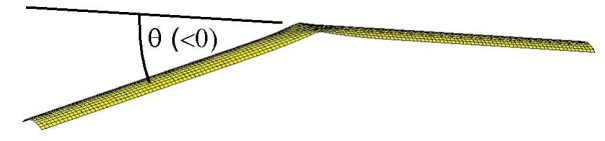

Fig. 1 Two Dimensional Tape Spring Fold

of hinge systems such as the Tape Spring Rolamite (TSR) hinge ${ }^{5}$ (developed by the Deployable Structures Lab (DSL) in Cambridge, UK) and the French low cost tape spring hinge ${ }^{6}$ (developed by CNES/METRAVIB RDS on behalf of the CNES/DEMETER programme). When a tape spring of length $\mathrm{L}$ is loaded by equal and opposite applied moments $\mathrm{M}$, in the soft plane of the tape spring, a standard moment-rotation relationship is produced as shown in figure 2. Buckling

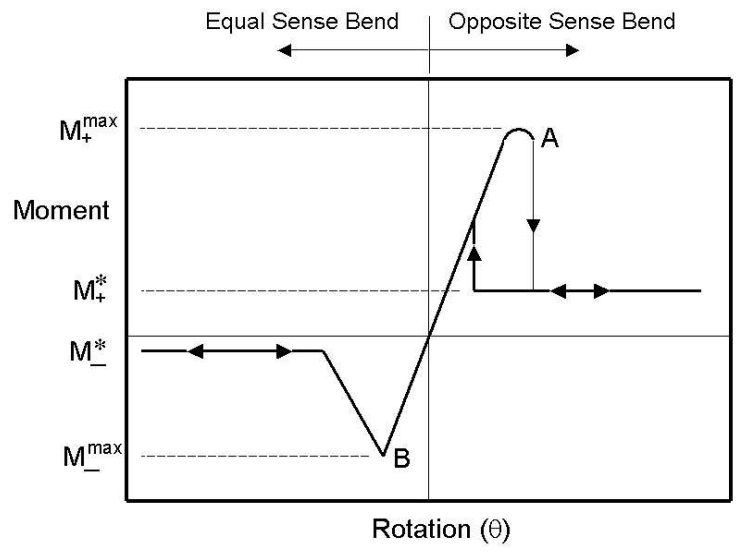

Fig. 2 Moment-Rotation relationship for a two dimensional tape spring fold

occurs at $\mathrm{A}$ and $\mathrm{B}$ (known as the peak moments), after which localized folds form in the tape spring. The reaction moments of the tape spring then remain approximately constant. These constant moments are known as steady state moments.

This moment-rotation relationship is the basis of two dimensional tape spring bend analysis, which attempts to predict the peak moments $\left(M_{+}^{\max }, M_{-}^{\max }\right)$ and the steady state moments $\left(M_{+}^{*}, M_{-}^{*}\right)$. It is defined that positive applied moments produce tension along the edges of the strip resulting in a longitudinal curve that is in the opposite sense to the initial transverse curve of the tape spring. This is known as an opposite sense fold. Conversely, negative applied moments create compression along the edges of the strip, resulting in equal sense folds. ${ }^{7}$ Opposite sense folds fail via 'snap through' buckling whereas equal sense bends fail via a torsional buckling mode. This means that opposite sense peak moments are higher than equal sense peak moments.

Three-dimensional tape spring folds not only incorporate longitudinal bending but also twisting of the section. The relative twist angle between the ends of the tape spring is denoted by $\gamma$. A three dimensional fold can be seen in figure 3. Such a fold is formed

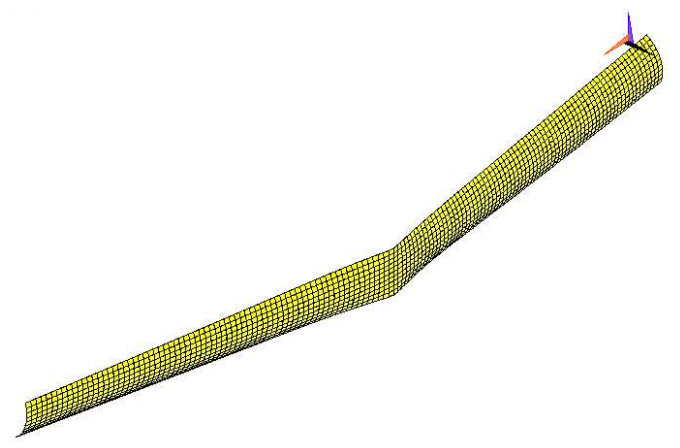

Fig. 3 Three Dimensional Tape Spring Fold $(\theta=$ $20^{\circ}, \gamma=40^{\circ}$ )

whenever the fold line is not perpendicular to the axis of the tape (see figure 4). Therefore, if the tape spring is not mounted perpendicular to the deployment appendage, a three dimensional fold will be created. It

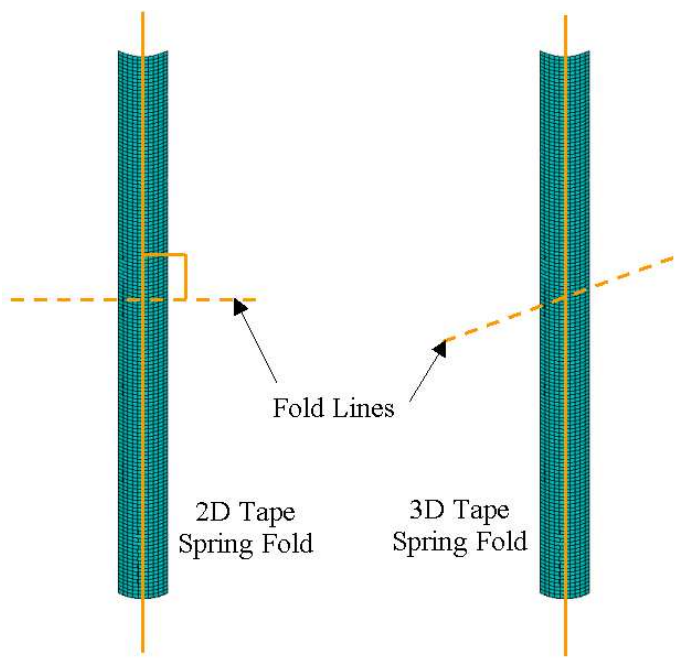

Fig. 4 Fold Line Overlayed on a Tape Spring 
should be noted that if the true fold line is at $45^{\circ}$ to the two dimensional fold line then a 'perfect' three dimensional fold is created. This results in a final fold of $\theta=90^{\circ}$ and $\gamma=180^{\circ}$. The 'perfect' fold motion, therefore, for all experimental and theoretical static tests follows the path defined by $\gamma=2 \theta$.

Future deployment designs have been presented in previous studies that incorporate three dimensional folds. ${ }^{8}$ There are specific advantages in using angled tape springs to deploy panels, such as a thinner packing thickness if the tape springs are mounted on the upper and lower panel surface (increasing the stiffness of the hinge). It is therefore necessary to perform some initial investigations into the deployment dynamics of such systems.

\section{Static Analysis of Three Dimensional Tape Spring Folds}

In-order to perform a theoretical analysis of the tape spring it is first necessary to define the co-ordinate system. Due to the nature of the displacement, two co-ordinate systems are required to describe the motion of the tape. These can be seen in figure 5. Axes

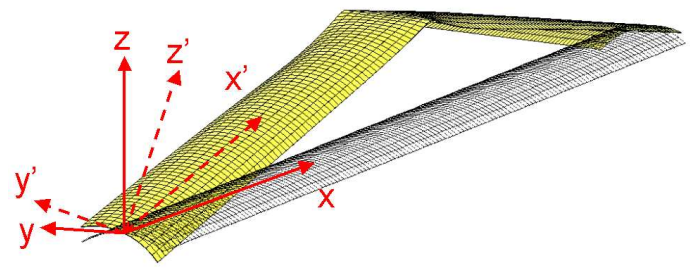

Fig. 5 The rotating co-ordinate system

$\mathrm{x}, \mathrm{y}$ and $\mathrm{z}$ are a fixed co-ordinate system in relation to the initial unfolded length of tape. The dashed axes shown denote a moving co-ordinate system which stays in the flat plane of the tape spring as shown. Longitudinal bending occurs around the $y$ axis and twisting occurs around the $\mathrm{x}$ ' axis.

From past research it is known that the momentrotation plot can be theoretically simulated using equation 1.

$\bar{M}=\bar{\kappa}_{x}-\bar{\kappa}_{x, 0}+\left\{\frac{\lambda}{\left(1-\nu^{2}\right)}\right\}\left\{\mu \Psi_{1}\left(\bar{\kappa}_{x}\right)-\lambda \bar{\kappa}_{x} \Psi_{2}\left(\bar{\kappa}_{x}\right)\right\}$

where:

$$
\begin{gathered}
\mu=2 \nu \bar{\kappa}_{x}-\bar{\kappa}_{y, 0}-\nu \bar{\kappa}_{x, 0} \\
\lambda=\bar{\kappa}_{x y}^{2}-\bar{\kappa}_{x y, 0}^{2}+\left(\bar{\kappa}_{x}-\bar{\kappa}_{x, 0}\right)\left(\nu \bar{\kappa}_{x}-\bar{\kappa}_{y, 0}\right) \\
\Psi_{1}\left(\bar{\kappa}_{x}\right)=\frac{1}{\bar{\kappa}_{x}^{2}}\left[1-\frac{1}{\bar{\kappa}_{x}^{\frac{1}{2}}}\left(\frac{\cosh \left(2 \bar{\kappa}_{x}^{\frac{1}{2}}\right)-\cos \left(2 \bar{\kappa}_{x}^{\frac{1}{2}}\right)}{\sinh \left(2 \bar{\kappa}_{x}^{\frac{1}{2}}\right)+\sin \left(2 \bar{\kappa}_{x}^{\frac{1}{2}}\right)}\right)\right]
\end{gathered}
$$

$$
\begin{aligned}
& \Psi_{2}\left(\bar{\kappa}_{x}\right)=\frac{1}{\bar{\kappa}_{x}^{4}}\left[1+\frac{\sinh \left(2 \bar{\kappa}_{x}^{\frac{1}{2}}\right) \sin \left(2 \bar{\kappa}_{x}^{\frac{1}{2}}\right)}{\left(\sinh \left(2 \bar{\kappa}_{x}^{\frac{1}{2}}\right)+\sin \left(2 \bar{\kappa}_{x}^{\frac{1}{2}}\right)\right)^{2}}\right. \\
& \left.-\frac{5}{4 \bar{\kappa}_{x}^{\frac{1}{2}}}\left(\frac{\cosh \left(2 \bar{\kappa}_{x}^{\frac{1}{2}}\right)-\cos \left(2 \bar{\kappa}^{\frac{1}{2}}\right)}{\sinh \left(2 \bar{\kappa}_{x}^{\frac{1}{2}}\right)+\sin \left(2 \bar{\kappa}_{x}^{\frac{1}{2}}\right)}\right)\right]
\end{aligned}
$$

This is the fundamental equation derived by Mansfield $(1973)^{9}$ using shell theory mathematics. Equation 1 is essentially a large equation that is simply $\bar{M}=f\left(\bar{\kappa}_{x}\right)$, which is the equivalent of $M=f(\theta)$. Non-dimensional values of the longitudinal moment and the curvatures are used (denoted by $\bar{M}$ and $\bar{\kappa}$ respectively) to avoid indeterminate expressions in the main equation. These non-dimensional parameters are found using equations 6 and 7.

$$
\bar{M}=\left(\frac{3 a\left\{3\left(1-\nu^{2}\right)\right\}^{\frac{1}{2}}}{E t^{4}}\right) M
$$

$$
\begin{aligned}
&\left\{\bar{\kappa}_{x, 0}, \bar{\kappa}_{x y, 0}, \quad \bar{\kappa}_{y, 0}, \bar{\kappa}_{x}, \bar{\kappa}_{x y}\right\}= \\
& \frac{a^{2}\left\{3\left(1-\nu^{2}\right)\right\}^{\frac{1}{2}}}{4 t}\left\{\kappa_{x, 0}, \kappa_{x y, 0}, \kappa_{y, 0}, \kappa_{x}, \kappa_{x y}\right\}
\end{aligned}
$$

There is an equivalent equation for the tape spring reaction torque, as shown in equation 8 . This is a function of two curvature variables, $\bar{T}=f\left(\overline{\kappa_{x}}, \overline{\kappa_{x y}}\right)$ which is the equivalent of $T=f(\theta, \gamma)$.

$$
\begin{gathered}
\bar{T}=\bar{\kappa}_{x y}-\bar{\kappa}_{x y, 0}+\frac{\lambda \bar{\kappa}_{x y} \Psi_{1}\left(\bar{\kappa}_{x}\right)}{1-\nu} \\
\bar{T}=\left(\frac{3 a\left\{3\left(1-\nu^{2}\right)\right\}^{\frac{1}{2}}}{4 G t^{4}}\right) T
\end{gathered}
$$

From mathematical studies of three dimensional surface curvatures it is known that a three dimensional surface is defined by two lines of principle curvature, which are perpendicular to each other. These are the lines of maximum and minimum curvature of the surface. If this is applied to the post buckling analysis of tape springs it can be seen that as twist is applied to the tape, the lines of principle curvature rotate (see figure 6 ). The angle of this rotation is signified by the change in $\phi$. This rotation of $\phi$ impacts the surface

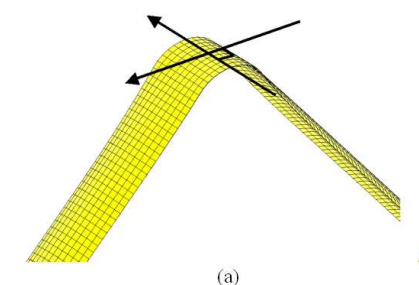

(a)

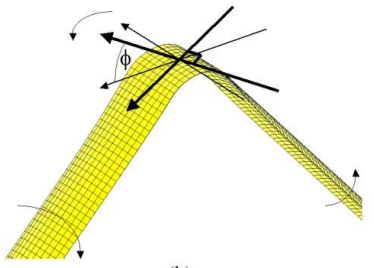

(b)
Fig. 6 The rotation of the lines of principle curvature

curvature matrix as shown in equation 10 , where $\lambda_{1}$ and $\lambda_{2}$ are the maximum and minimum curvatures of the surface respectively.

$\kappa=\left(\begin{array}{cc}\cos \phi & \sin \phi \\ -\sin \phi & \cos \phi\end{array}\right)\left(\begin{array}{cc}\lambda_{1} & 0 \\ 0 & \lambda_{2}\end{array}\right)\left(\begin{array}{cc}\cos \phi & -\sin \phi \\ \sin \phi & \cos \phi\end{array}\right)$ 
Therefore as the twist changes, assuming constant values for $\lambda_{1}$ and $\lambda_{2}, \kappa_{x}$ can be recalculated. Using equations 7,1 and 6 , a value for the longitudinal reaction moment can then be found. This solution, however, does not take into account of the length of the tape spring (hence the resultant rotation of the flat strips either side of the central fold). In-order to include this into the analysis, the theoretical tape model was divided into three sections as shown in figure 7 . Due

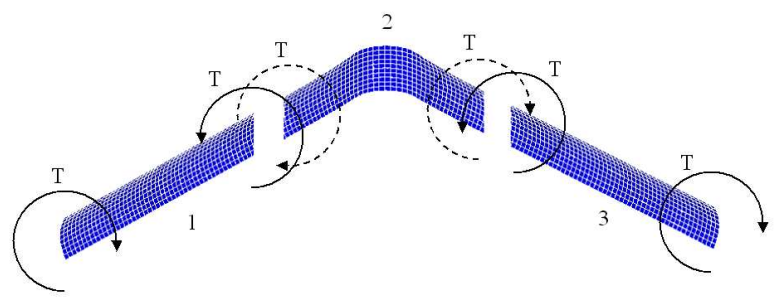

Fig. 7 Torque balance of tape spring sections

to the symmetry of the model, sections 1 and 3 are assumed to be identical. The twist of these sections are calculated using standard torsion theory. ${ }^{10}$ The resultant torque from these sections is balanced with the reaction torque from section 2. This calculation outputs the resultant rotation of the principle curvature lines, which in turn allows the longitudinal and twisting reaction moments to be calculated.

This theory was implemented with the parameters shown in table 1 and compared with a experimental test results of 'perfect' three dimensional tape spring bends. (The experimental test rig is shown in figure 8.) The analytical results, overlayed on the experimental

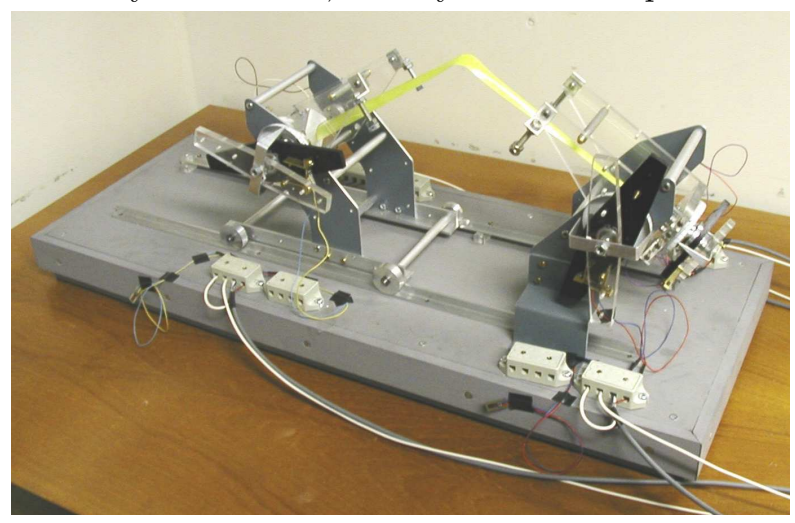

Fig. 8 Experimental Test Rig

results can be seen in figure 9 (for the longitudinal moment $\mathrm{M}$ ), and figure 10 (for the twisting moment $\mathrm{T}$ ). It was found that, if two critical tape parameters (the torsional stiffness of sections 1 and 3, and the length of section 2) are accurately determined then the theoretical model would closely model the experimental result. It was found and confirmed that the presence of an incrementing twist angle in the fold causes the longitudinal steady state moment to rise. This, in turn, would have an impact on the deployment dynamics of $3 \mathrm{D}$ bends compared to $2 \mathrm{D}$ bends.

\begin{tabular}{|c|l|}
\hline $\mathrm{E}$ & $205 \times 10^{3} \mathrm{Nmm}$ \\
\hline$\nu$ & 0.3 \\
\hline$\alpha$ & $1.25 \mathrm{rad}$ \\
\hline $\mathrm{t}$ & $0.11 \mathrm{~mm}$ \\
\hline$R_{y}$ & $20 \mathrm{~mm}$ \\
\hline$\kappa_{y, 0}$ & 0.05 \\
\hline$\kappa_{x, 0}$ & 0 \\
\hline $\mathrm{D}$ & $24.99 \mathrm{Nmm}^{4}$ \\
\hline $\mathrm{L}$ & $400 \mathrm{~mm}^{4}$ \\
\hline
\end{tabular}

Table 1 Properties of experimental tape spring cross-section

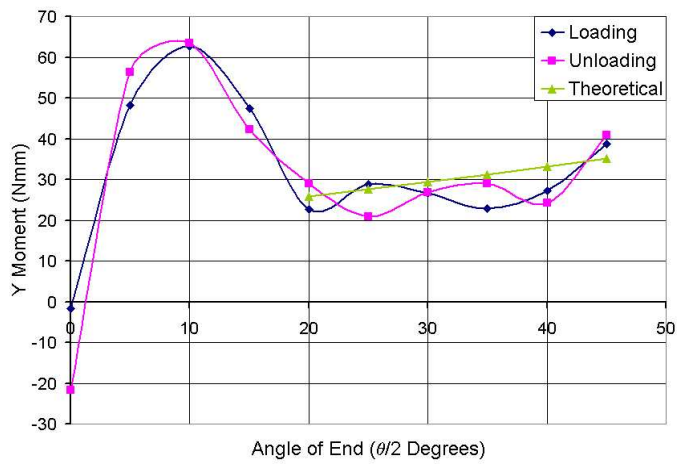

Fig. 9 Longitudinal Reaction Moment Results

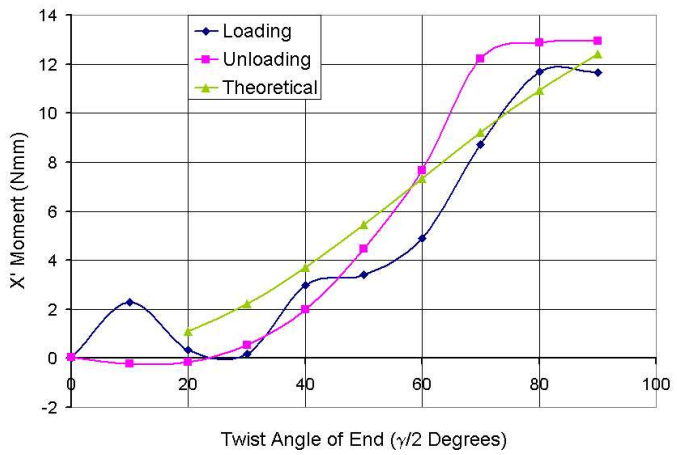

Fig. 10 Twisting Reaction Moment Results

\section{Initial Dynamic Model Predictions}

Dynamic modelling of two dimensional tape springs have been previously performed in-order to study the deployment dynamics of simple composite panels. ${ }^{11}$ The full model for the deployment of an equal sense, two dimensional tape spring fold is based on the modelled moment rotation plot shown in figure 11, where the co-ordinates of the critical points can be calculated from both analytical expressions (derived by Wüst $(1954)^{12}$ and Rimrott $(1966)^{13}$ ) and polynomial functions (derived by Seffen $(1998)^{11}$ ) within reasonable accuracy limits.

In this current work the tape is modelled as a 'simple hinge' in order to study the impact of the steady state moment rise on the system dynamics, as this is the largest time segment of the tape spring deployment (especially if $\theta(@ t=0)=\pi$ ). Only 'perfect' bends will be studied as this is the 'worst case' for the impact of 


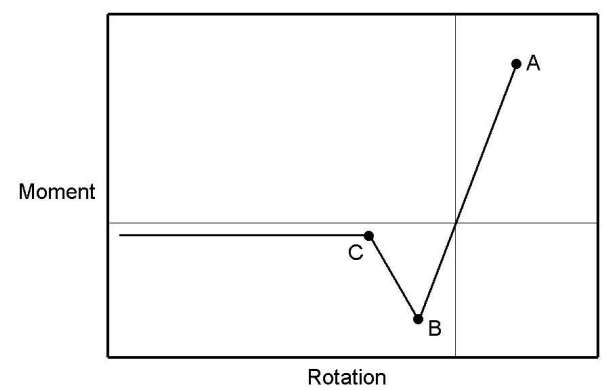

Fig. 11 Moment rotation plot for 2D Equal Sense Bend Deployment

twist on the longitudinal moment. The pre-buckling moment-rotation relationship is assumed to be equivalent to the two dimensional model due to the relative small angles of $\theta$ involved.

By linearly interpolating the result of the post buckling analytical model, a simple relationship was found between $\mathrm{M}$ and $\theta$ (for $\theta>14.6^{\circ}$ ), shown in equation 11 , where $\theta$ is measured in radians, $m_{A}=0.0102$ and $m_{B}=0.0188$.

$$
\begin{gathered}
M=m_{A} \theta+m_{B} \\
M=I_{y} \ddot{\theta}
\end{gathered}
$$

A simple dynamic model ${ }^{10}$ can then be created by substituting equation 11 into 12 . The moments of inertia about the $\mathrm{x}$ ' and $\mathrm{y}$ axes were calculated as a number of small lumped masses, at increasing lengths from the fold point. Equation 12 can then be rearranged into equation 13 , which is the standard format for a linear, second order, differential equation with known constant coefficients $\left(m_{C}=\frac{m_{A}}{I y}\right.$ and $\left.m_{D}=\frac{m_{B}}{I y}\right)$ and no damping.

$$
\ddot{\theta}+m_{C} \theta=-m_{D}
$$

Solving equation 13 with the boundary conditions $\theta(0)=\frac{\pi}{2}$ and $\dot{\theta}(0)=0$ results in the general solution shown in equation 14 .

$$
\theta=A_{m} \cos \left(\lambda_{i} t\right)+B_{m} \sin \left(\lambda_{i} t\right)+C_{m}
$$

where:

$$
\begin{gathered}
\lambda_{i}=\sqrt{m_{C}} ; \quad C_{m}=-\frac{m_{D}}{m_{C}} ; \\
B_{m}=0 ; \quad A_{m}=\frac{\pi}{2}-C_{m}
\end{gathered}
$$

The overall solution can be seen in figure 12 (denoted by $\theta 1$ ), overlayed on the two dimensional result (denoted by $\theta 2$ ), with a steady state moment of $-21.86 \mathrm{Nmm}$. It can be seen that the steady state moment rise has shortened the deployment time by $18 \%$, and from figure 13 the angular velocity before snap back (point C, figure 11) has increased by $13.5 \%$.

Referring back to figure 12 it can be seen that there is also an overlay of $\gamma$ for the twisting dynamics of the deployment. This is calculated using a similar expression as equation 11 but without the constant term (as zero twist results in zero reaction torque) where $m_{A}$ $=0.0036$. The two independently calculated dynamic

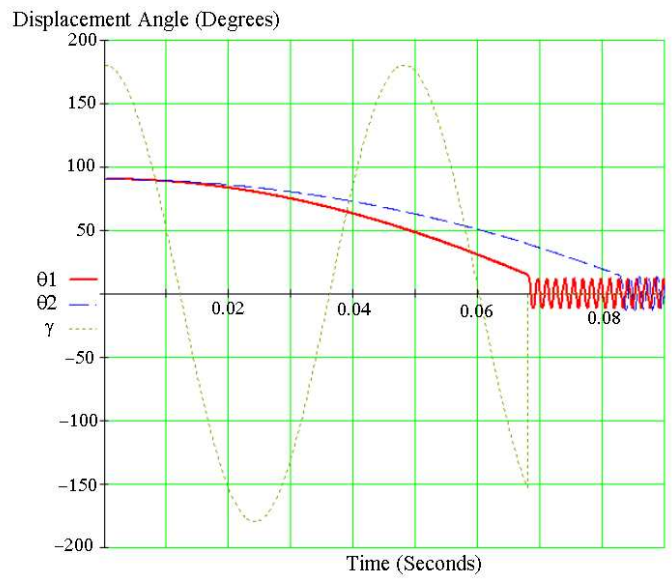

Fig. 12 Displacement Deployment Model of Tape Spring

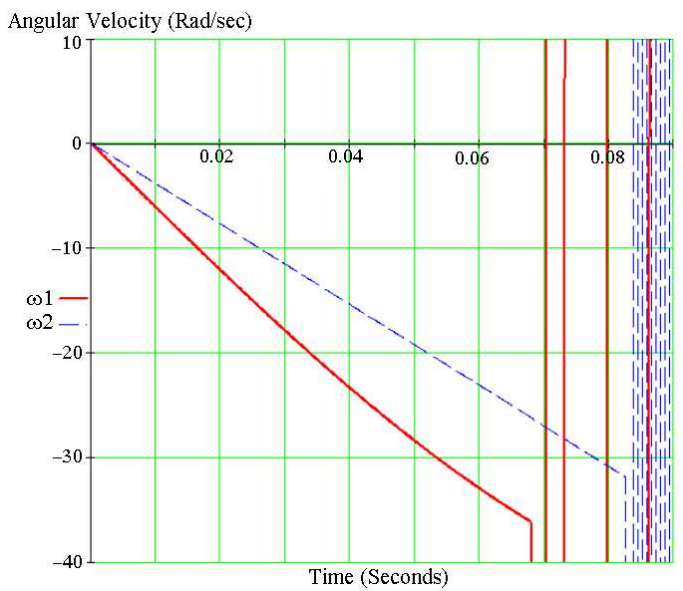

Fig. 13 Angular Velocity Deployment Model of Tape Spring

results $(\theta 1$ and $\gamma)$ suggest that, due to the low inertia of the tape about the $\mathrm{x}$ ' axis, the tape twists by just under 1.5 oscillations before the 'snap back'. For an undamped, theoretical model of a tape spring released in free space (as shown previously in figure 3) this may be a representative result. However, from experimental testing it is clear that for any practical purpose, (involving tape spring fixings and attachments) this does not occur.

If a more realistic system is considered (shown in figure 14), where one end of the tape spring is fixed in space, and the other is moved to form a 'perfect' $\left(\theta=90^{\circ}, \gamma=180^{\circ}\right)$ three dimensional fold, then from experimental observations, the nature of the dynamics is very different from the perfect theory. It can be concluded from experimental observations that there are three primary stages for the deployment of a three dimensional fold in this configuration. The first stage, once released is the rapid twist correction of the free half of the spring, with little comparative oscillations. This is due to the fact that oscillations beyond this zero twist point would result in high twist reaction mo- 


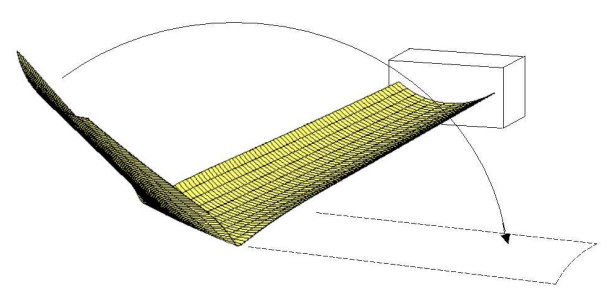

Fig. 14 'Realistic' system layout

ments from the fold (currently twisted in the opposite sense). The second stage is the longitudinal unfolding of the tape spring. Any twist remaining is over the tape length between the fold and the fixed end. The impact of this on the longitudinal deployment is related to the way the tape is fixed and the length of the tape from the fixed end to the fold. If the fold is well outside the region of end effects (created by the fixed end) then as $\theta$ reduces the fold will move towards the fixed end. The final stage is the low $\theta$ snap back, followed by twist oscillations. If the momentum of the spring is large enough, the tape spring 'bounces' out and buckles the tape in the opposite direction of twist. Note that this is the equivalent of the two dimensional fold 'snap through', which is a sign change in $\theta$, as apposed to this three dimensional case which is a sign change in $\gamma$.

\section{Dynamics of Array Deployment}

The true deployment nature of tape springs folded in three dimensions is clearly very complex and very specific to the system in which they are incorporated. Therefore to proceed further it is necessary to apply the dynamic models to a specific deployment system. Further analysis will focus on the stage two deployment of a simply supported area deployment device (as presented in Walker and Aglietti $(2002)^{8}$ ). This design incorporates two, three dimensionally folded tape springs which deploy to support an array fabric as shown in figure 15 (where the thick dotted lines denote the tape springs). To model the deployment of

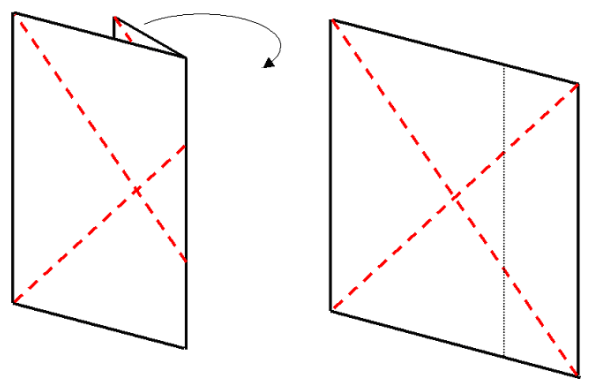

Fig. 15 Area deployment

this area it is first necessary to determine the 'hinge' moment about the area fold line. This 'hinge' moment is determined by resolving the longitudinal and twisting moments in the tape spring. All cross coupling of the resolved moments is ignored as this analysis is simply repeatedly used to determine 'snap' shots of the moments in the system. It is first necessary to define some variables used in the analysis.

The three critical angles in this analysis are $\mu, \Delta$ and $\beta$. $\mu$, which is the angle the tape spring is mounted away from the perpendicular of the fold line, can be seen in figure 16. $\Delta$, which is the angle of the array from the initial position, is shown in figure 17. (This figure also defines $\Lambda$ which is the array angle used in the dynamic analysis.) The angle $\beta$, which is the angle of the plane (that contains both halves of the tape spring) from the horizontal, is shown in figure 18. The

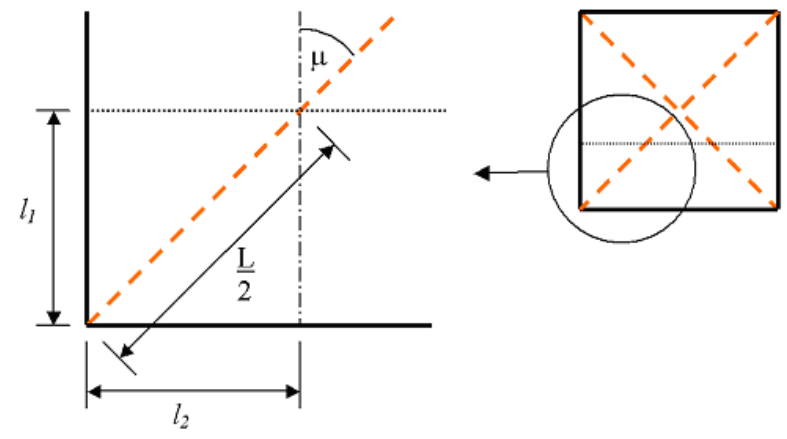

Fig. 16 Variables for Analysis 1

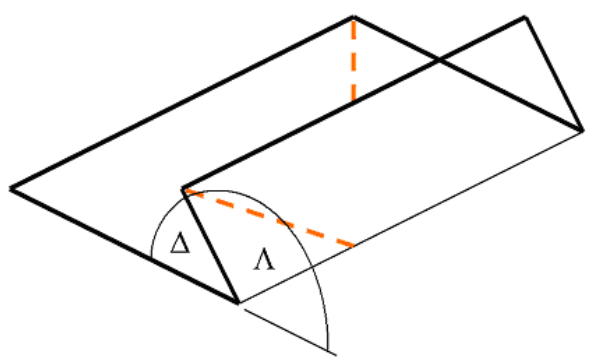

Fig. 17 Variables for Analysis 2

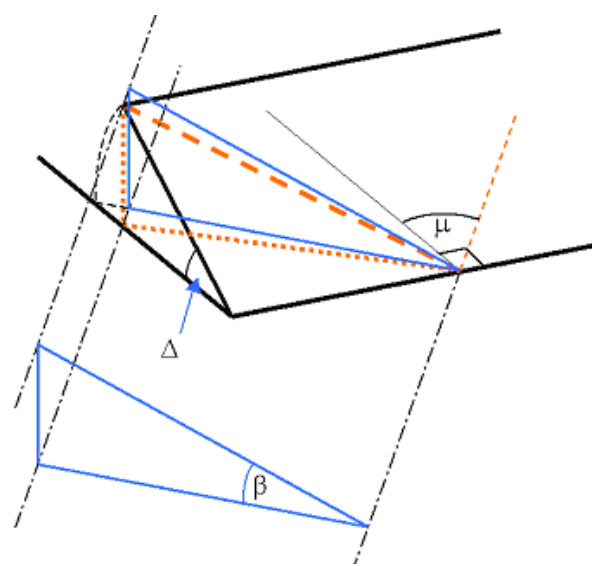

Fig. 18 Variables for Analysis 3

'hinge' moment around the fold line is then the sum of the resolved twist and longitudinal moments where the longitudinal moment is a function of $\theta$ and the twist 
moment is a function of $\gamma$ (as shown in equation 16).

$$
M_{\text {hinge }}=M(\theta) \sin \beta \cos \mu+T(\gamma) \sin \mu
$$

$\gamma$ can be simply determined using the relationship $\gamma=\pi-\Delta$. However, in order to determine $\theta$ it is first necessary to determine the angle $\beta$ in terms of $\Delta$ and $\mu$. This has to be performed separately for $\Delta \leq \frac{\pi}{2}$ and $\Delta \geq \frac{\pi}{2}$.

For $\Delta \leq \frac{\pi}{2}$ :

$$
\beta=\tan ^{-1}\left\{\frac{l_{1} \sin \Delta}{l_{2} \cos \mu+l_{1} \cos \Delta \sin \mu}\right\}
$$

For $\Delta \geq \frac{\pi}{2}$ if

$$
\varepsilon=\Delta-\frac{\pi}{2}
$$

then

$$
\beta=\tan ^{-1}\left\{\frac{l_{1} \cos \varepsilon}{l_{2} \cos \mu-l_{1} \sin \varepsilon \sin \mu}\right\}
$$

If $\mu \geq \frac{\pi}{4}$ then $\theta$ can be found using equation 21 .

$$
\theta=\sin ^{-1}\left\{\frac{2\left(l_{2} \cos \mu-l_{1} \sin \varepsilon \sin \mu\right)}{L \cos \beta}\right\}
$$

If, however, $\mu \leq \frac{\pi}{4}$ (i.e. $\theta$ in the un-deployed configuration is greater than $\frac{\pi}{2}$ ) then initially $\sigma$ must be determined before $\theta$ can be calculated. ( $\sigma$ is the internal angle between both longitudinal halves of the tape spring.) This method is used until $\sigma=\frac{\pi}{2}$, after which $\theta$ can then be calculated directly using equation 21 .

So if $\mu \leq \frac{\pi}{4}$ and $\sigma \leq \frac{\pi}{2}$ then:

$$
\sigma=\sin ^{-1}\left\{\frac{2\left(l_{2} \cos \mu-l_{1} \sin \varepsilon \sin \mu\right)}{L \cos \beta}\right\}
$$

Therefore:

$$
\theta=\pi-\sigma
$$

The values for this analysis are shown in table 2 .

\begin{tabular}{|c|l|}
\hline $\mathrm{L}$ & $0.8485 \mathrm{~m}$ \\
\hline$l_{1}, l_{2}$ & $0.3 \mathrm{~m}$ \\
\hline$\mu$ & $\frac{\pi}{4} \mathrm{rad}$ \\
\hline$I_{\text {array }}$ & $9.525 \times 10^{-3} \mathrm{kgm}^{2}$ \\
\hline
\end{tabular}

Table 2 Properties of array

$M_{\text {hinge }}$ was then determined for the deployment using the static analysis method and equations 16, 17, 20 and 21 . This resulted in a moment rotation relationship up to $\theta=14.6^{\circ}$ as shown in figure 19 . It can be seen that the initial hinge moment (dominated by the torque) is low. It also displays that the moment magnitude throughout the deployment is lower that the steady state moment for a two dimensional fold. Using the inertial properties of the array, a dynamic

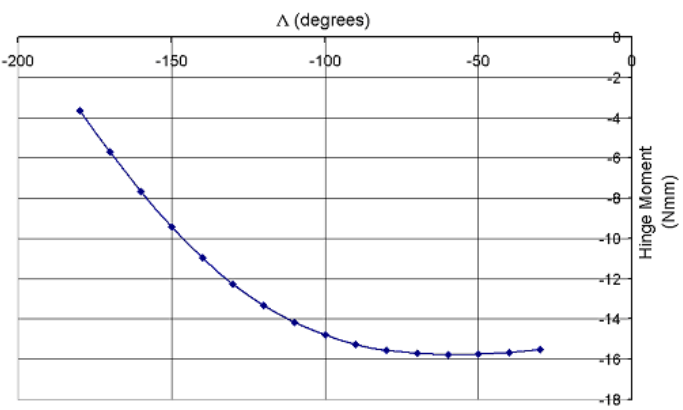

Fig. 19 Moment-Rotation Relationship for Array

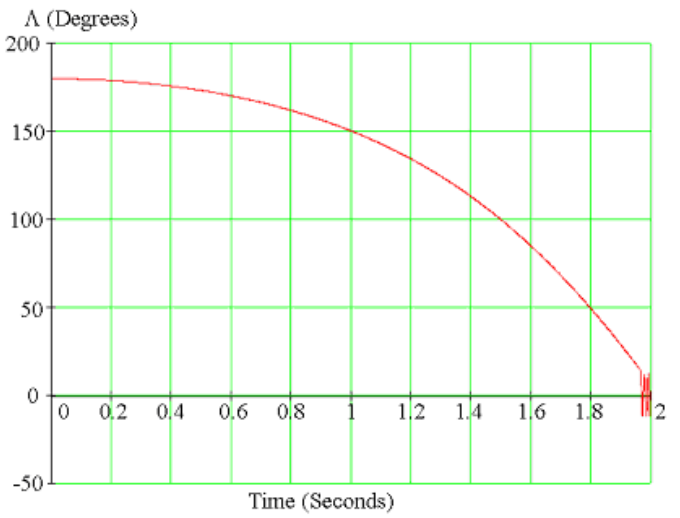

Fig. 20 Displacement Deployment Model of Array

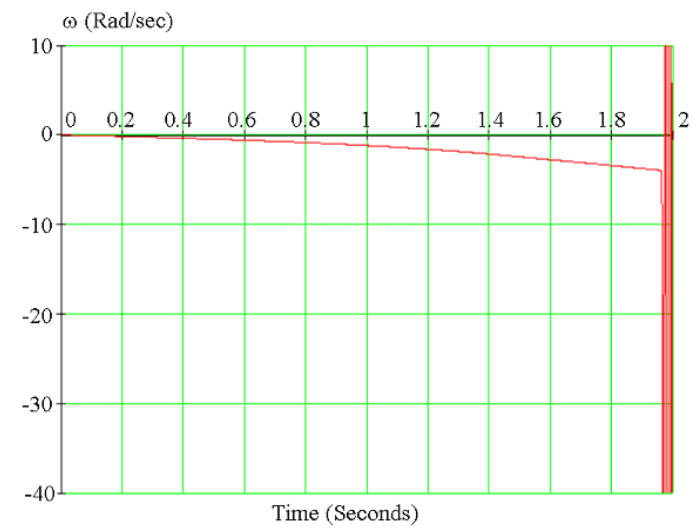

Fig. 21 Angular Velocity Deployment Model of Array

model was created. The displacement dynamics and angular velocity models are shown in figures 20 and 21 respectively. It can be seen that (without damping) the deployment time has increased to just under two seconds and the angular velocity just before snap back is much lower than the free tape spring, as expected. By applying an energy method (used previously ${ }^{11}$ ) a prediction can be made as to whether the tape spring will snap through, without damping, forming an opposite sense fold. The method compares the area under the moment-rotation curve to the left of the vertical axis with the area under the curve on the right of the vertical axis. Figure 22 displays the equivalent two dimensional system. It was found that the unfold- 


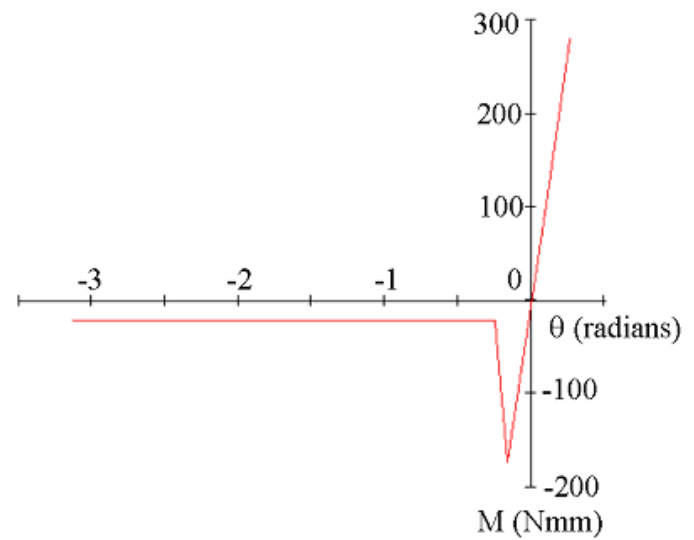

Fig. 22 Full Moment Rotation Plot for 2D Tape Spring Fold

ing energy of the two dimensional system was 2.368 times greater than the threshold energy necessary to cause 'snap through'. The equivalent three dimensional system (shown in figure 23) shows that (due to the low initial moments about the hinge) the deployment energy has reduced significantly to 1.678 times the threshold energy. Although a 'snap through' is predicted to occur the energy available to cause a 'snap though' has been halved. Therefore, not only is the

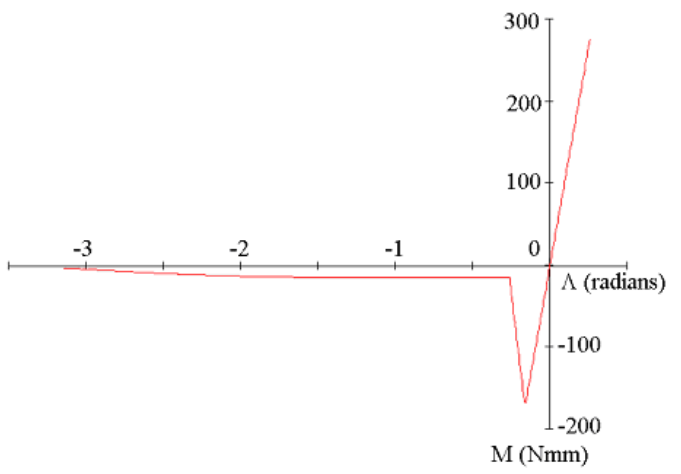

Fig. 23 Full Moment Rotation Plot for 3D Tape Spring Fold

deployment slower, but also less damping is required in the system to prevent 'snap through'.

\section{Conclusions}

This work has focused on the deployment dynamics of three dimensional tape spring folds, by initially studying the static nature of the forces involved then applying them to a specific area deployment application. The static reaction moments produced by a three dimensional tape spring fold can be determined using shell theory mathematics, three dimensional surface mathematics and standard torsion theory. Using simple dynamic models the undamped dynamic nature of a tape spring in free space could easily be determined. By deriving a set of equations to determine the impact of the tape spring moments on the hinge or fold line of an array, these methods could then applied to a specific deployment application. It was found that, compared to the two dimensional case, the deployment was slower (reaching much lower angular velocities) and the deployment energy of the system was significantly reduced, make it easier to prevent a snap through of the tape springs. This, in turn, would reduce the damping required in the system, and hence reduce the mass of the array.

\section{References}

${ }^{1}$ Bishop, D., Giles, R., and Austin, G., "The Lucent LambdaRouter: MEMS technology of the future here today," IEEE Communications Magazine, Vol. 40, No. 3, 2002, pp. 75-79.

${ }^{2}$ Paine, M. and Gabriel, S., "A micro-fabricated colloidal thruster array," AIAA paper, 2001, AIAA 2001-3329.

${ }^{3}$ Simburger, E., Matsumoto, J., Lin, J., Knoll, C., Rawal, S., Perry, A., Barnett, D., Peterson, T., Kerslake, T., and Curtis, H., "Development of a Multifunctional Inflatable Structure for the Powersphere Concept," 43rd AIAA/ASME/ASCE/AHS Structures, Structural Dynamics and Materials Conference, 2002, AIAA 2002-1707.

${ }^{4}$ Seffen, K. A., You, Z., and Pellegrino, S., "Folding and deployment of curved tape springs," International Journal of Mechanical Sciences, Vol. 42, 2000, pp. 2055-2073.

${ }^{5}$ Watt, A. and Pellegrino, S., "Tape-Spring Rolling Hinges," Proceedings of the 36th Aerospace Mechanisms Symposium, Glenn Research Center, 2002.

${ }^{6}$ Givois, D., Sicre, J., and Mazoyer, T., "A Low Cost Hinge for Appendices Deployment: Design, Test and Applications," 9th European Space Mechanisms and Tribology Symposium ESA/ESTEC, 2001.

${ }^{7}$ Seffen, K. A. and Pellegrino, S., "Deployment dynamics of tape springs," Proc. Roy. Soc. Lond., Vol. A 455, 1999, pp. 1003-1048.

${ }^{8}$ Walker, S. and Aglietti, G., "An application of Tape Springs in Small Satellite Area Deployment Devices," Proceedings of 5th International Conference on Dynamics and Control of Systems and Structures in Space, 2002.

${ }^{9}$ Mansfield, E. H., "Large-deflection torsion and flexure of initially curved strips," Proc. Roy. Soc. Lond., Vol. A. 334, 1973, pp. 279-298.

${ }^{10}$ Megson, T., Aircraft Structures for Engineering Students (Second Edition), Edward Arnold, 1990.

${ }^{11}$ Seffen, K. A., Pellegrino, S., and Parks, G., "Deployment of a Panel by Tape-Spring Hinges," Proceedings of IUTAM-IASS Symposium on Deployable Structures: Theory and Applications, 1998, pp. 355-364.

${ }^{12}$ Wüst, W., "Einige Andvendungen der Theorie der Zylinderschale," Z. Angew. Math. Mech., Vol. 34, 1954, pp. 444-454.

${ }^{13}$ Rimrott, F., "Storable Tubular Extendible Members," Engineering Digest, 1966. 